\title{
A Generic Approach of ECG Signals Classification Using CNN
}

\author{
${ }^{1}$ P. Rama Santosh Naidu, ${ }^{2}$ Dr. G. Lavanya Devi \\ ${ }^{1}$ Department of Computer Science and Engineering, MVGR College of Engineering (A), Andhra Pradesh, India \\ prsn1988@gmail.com \\ ${ }^{2}$ Department of Computer Science and Systems Engineering, Andhra University College of Engineering (A), \\ Andhra Pradesh, India \\ lavanyadevig@yahoo.co.in
}

Received: 09th September 2020, Accepted: 14th October 2020, Published: 31st October 2020

\begin{abstract}
Based on current medical applications, classification of The ECG signs performs a crucial role in the diagnosis of heart disease. The challenging part is to obtain an accurate classification of ECG. There are Machine learning algorithms available that provide solutions for classification as well as feature extraction of ECG data available. But these solutions only confine to basic feature learning architectures but not in-depth extraction. ANN classifiers are one such mainstream classifier widely utilized in the relegation of ECG signals. In this paper, a versatile 1-D Convolutional Neural Network $(\mathrm{CNN})$ is used for feature extraction and classification performed on two datasets (MIT-BIH and PTBDB) which are presented in the result section. Visualized 5000 sampled data points using the embeddings of our baseline CNNs. Here we show the visualization obtained from the application of PCA to extract the 2 most informative dimensions from these embeddings. We also use K-means to perform clustering, showing the results in the same representation. The proposed method is efficient and generic due to its parameter invariant nature which makes it applicable for any ECG dataset.
\end{abstract}

Keywords

CNN, K-Means, PCA

\section{Introduction}

The ECG sign is due to the ionic present-day waft, which reasons the cardiac fibers to agreement and relax and hence generates a time-variant periodic sign wherein the ability differentiation between the 2 electrodes mounted on the floor of the skin is believed to be an enter to the ECG plotter.

The electrical activities of the different human organs are represented by bioelectrical signal where Electrocardiograph (ECG) is one of the bioelectrical signals originated from the heart. Neural networks are one of the most effective pattern recognition techniques due to their high nonlinear complexity and their ability to decrease error in test inputs by responding to the input response pattern. The selection index is one of the most important activities for signal processing.

For this work, the MIT-BIH from Physionet's MIT-BIH Arrhythmia Dataset and PTBDB dataset from Physionet's PTB Diagnostic Database are classified through PCA, T-SNE and K-means on each dataset and represent them visually in the below sections.

PCA (Principal Component Analysis):

In short, PCA tries to find the principal components of the data, so it's looking for the directions where the data is more spread out, so where there is most variance. The first main component widespread, simple system gaining knowledge of set of rules that is educated on a take a look at facts set and explains maximum of the version inside the facts, the second one issue explains maximum of the variation, and so on. We will select the most important principal components and use them for clustering.

A PCA converts the correlations among of all the cells into a 2-D graph as shown in the below figure. Cells that are highly corelated are clustered together.

Here's one last main conception about how to interpret PCA plots:

The axes are graded in order of accuracy.

Differences along the first main component axis (PC1) are more significant than differences along the second main component axis (PC2). 


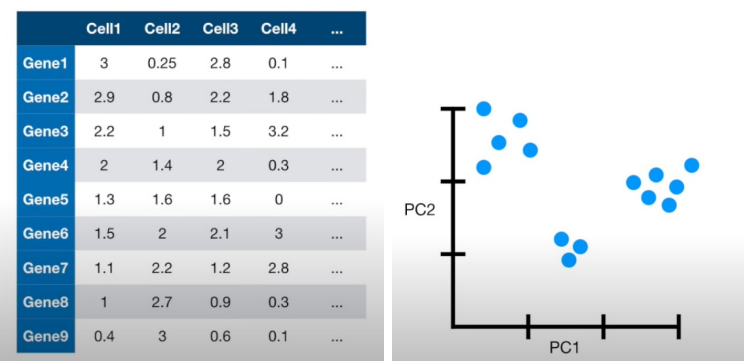

Figure 1: Example Demonstrating PCA

T-SNE (T-distributed Stochastic Neighborhood Embedding):

$\checkmark$ State of art/best dimensionality reduction technique for visualization of data.

$\checkmark$ It is graph-based, nonlinear and stochastic method.

$\checkmark$ It only preserves the local distance between the cells, so the distance between the groups are not meaningful.

$\checkmark$ It can run on top of principal components, which is how we use it and there are many parameters to optimize.

Example: From 2D to $1 \mathrm{D}$

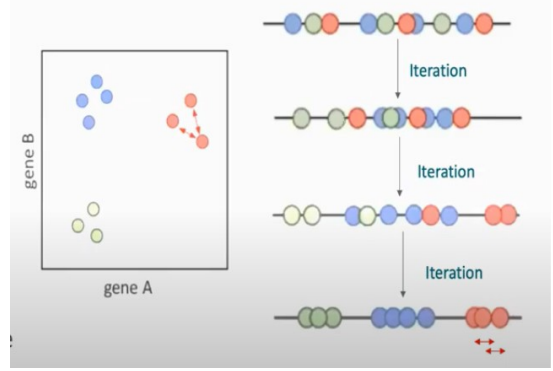

Figure 2: Example of Going 2D Data to 1D Data Using t-SNE

So basically, we are looking the distances between the different dots in the above figure and throughout iteration rounds, we try to make the data so that distances, local distances, are preserved in $2 \mathrm{D}$ model as shown the figure, so they are similar as the distances sin 1D model in the above figure. Remember the global distance, so for example the distance between the green and red group, doesn't mean that much, but the distance within the group is meaningful.

K-MEANS:

K-means algorithm is a standard, simple machine learning algorithm that is trained on a test data set and capable of relegating an incipient data set using $\mathrm{k}$ number of predefined clusters.

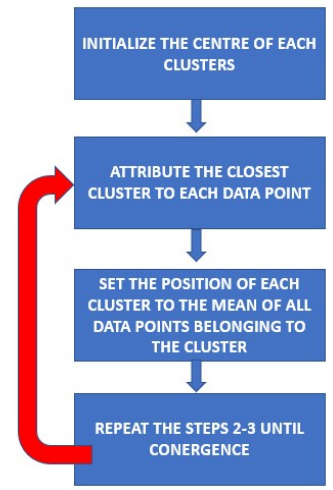

Figure 3: K-Means Algorithm Flowchart

The repositories (MIT-BIH \& PTBDB) encompass a huge variety of CSV files. every of those CSV files includes a matrix, and every row represents an instance inside the dataset section. The very last detail in each row is the elegance to which the instance belongs.

This dataset changed into used to investigate the detection of coronary heart beats using deep neural community models and to study some of the studying switch abilities. The impulses relate to the electrocardiogram (ECG) form of the heart beat in the normal case inside the cases of a couple of arrhythmias in myocardial infarctions. those indicators are pre-processed and segmented, corresponding to the heart beat of every part. 
MIT-BIH Arrhythmia Dataset:

Number of Categories: 5

Number of Samples: 109446

Sampling Frequency: $125 \mathrm{~Hz}$

Data Source: Physionet's MIT-BIH Arrhythmia Dataset

Classes: ['N': 0, 'S': 1, 'V': 2, 'F': 3, 'Q': 4]

The PTB Diagnostic ECG Database:

Number of Samples: 14552

Number of Categories: 2 (Normal vs Abnormal)

Sampling Frequency: $125 \mathrm{~Hz}$

Data Source: Physionet's PTB Diagnostic Database

\section{Background and Related Work}

Electrocardiogram (ECG) classification of signals plays a major role in the detection of the functioning of the heart. [1] This work involves the ECG signals, where the classifier is established for the detection of normal or pathological disorders of the heart. Raw ECG signals are obtained for classification from an online database (www.physioNet.org). The original ECG signal is pre-processed for noise reduction and the frequency range is analyzed to compare the original and denoised ECG signals. The attributes (P, Q, R, S, T time intervals) of the denoised ECG signal are analyzed and categorized using the Convolution Neural Network (CNN). The paper reports a classification strategy to separate ECG signals from the MIT-BIH database (arrhythmia database, pwave arrhythmia annotations, atrial fibrillation). CNN analyses the variance between the nominal set of parameters (amplitude and time interval) and classifies between the abnormality and the normal ECG pulse. This study provides a straightforward framework for interpreting the state of the clinician relevant to ECG and helps medical practitioners make informed decisions.

Even though convolutional neural networks (CNNs) may be used to categorize electrocardiogram (ECG) beats in cardiovascular ailment diagnostics, ECG indicators are conventionally regarded as unidimensional signals whereas CNNs are higher proper for multidimensional pattern or picture apperception applications.[2] in this analysis, the structure and rhythm state-of-the-art the heartbeats are amalgamated into a -dimensional information vector for next $\mathrm{CNN}$ analysis, which incorporates a most effective cutting-edge sample and an inequity cutting-edge drop-out technique. The findings monitor that the CNN model is rich in identifying eccentric heartbeats or arrhythmias by way of designates today's computerized extraction. as the proposed version become tested at the MIT-BIH arrhythmia index, the version completed higher reliability than other state-of-the-art techniques for 5 and 8 heart beat training (common accuracy ultra-modern 99.1 consistent with cent and 97\%). In truth, the machine used had improved sensitivity and wonderful predictive rates for $\mathrm{V}$ beats cutting-edge more than 4.3 per cent and 5.4 per cent. The framework ought to be comprehensive for implementation on mobile devices to manipulate cardiovascular disease at domestic.

Heart issues have been one in all the largest demanding situations dealing with public health for many years. according to the world fitness enterprise, ischaemic heart ailment is one of the pinnacle 10 leading causes of death.[3] automated analysis of aberrant heart assaults could also have the desired hospitalization for patients with coronary heart disorder, making it viable for them to shop their lives. Within the modern publish, we are making use of a streamlined however efficient deep mastering algorithm that focuses on easy convolutional units and time frequency presentations. The version will distinguish between three types of Electrocardiogram (ECG) alerts associated with three wonderful conditions, inclusive of sufferers with Congestive heart Failure, sufferers with Arrhythmia and sufferers with common heart beats. The 162 recordings used in the set of rules had been taken from the Physio net database, 96 from the Massachusetts Institute of technology-Beth Israel clinic (MIT-BIH) arrhythmia database, 36 from the usual MIT-BIH sinus rhythm database, and 30 from the Beth Israel Deaconess clinical center (BIDMC) for congestive coronary heart failure. The multiplicity and durability of the proposed technique will represent an actual $93.75 \%$ accuracy gain for actual-time surveillance. Recently, with the ostensible ascend in the range of coronary issues, ECG's computerized detection look at has played a main position in the scientific prognosis of cardiovascular disease.[4] The technique of the 1D convolution neural community $(\mathrm{CNN})$ is to cut up the ECG alerts. The CNN version includes five layers inserted into the enter layer and the output layer, i.e. convolution layers, two down sampling layers and one consummate connexon layer, placing apart the individual features from the pristine facts and mechanically relinquishing the features. The model recognizes the relegation of 5 normal varieties of arrhythmia signals, i.e., traditional, left package deal branch node, right package deal. The experimental outcomes of most of the people MIT-BIH cardiac arrhythmia data recommend that the tool achieves a promising relegation accuracy of 97.5 in line with cent, manner over maximum trendy graph relegation strategies. 


\section{Proposed Approach}

We visualize 5000 sampled data points using our baseline CNNs embeddings. Here we present the analysis obtained from the application of PCA to derive the 2 most important dimensions from these embeddings. We often use K-means to preform clustering, to display effects within the same representation. There is a pleasant class distinction in the MITBIH dataset, with some correlation between "Normal" and "S" and clustering being able to easily distinguish the "Q" and "V" classes. There is a bit of doubt in the

"Normal" class, possibly due to the vast majority of the dataset covered by the class. For the PTBDB dataset, the differentiation is not as compelling, with a significant overlap in visualization and a total loss in clustering. This suggests that the PTBDB dataset could be more difficult to identify. Further techniques, including T-sne, and raw data work are visualized as shown above in the graphical format.

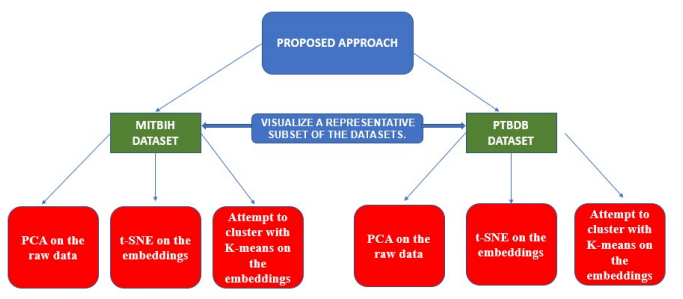

Figure 4: Diagrammatic Representation of Proposed Approach

\section{Results and Analysis}

FOR MITBIH DATASET:

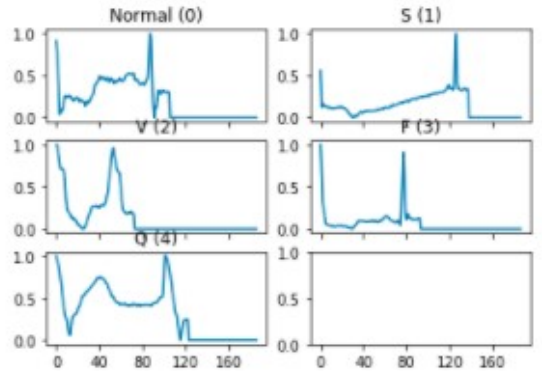

Graph 1: Plot One of Each Label - MITBIH Dataset

'N': NORMAL, 'S': supraventricular ectopic beat, ' $V$ ': ventricular ectopic beat , 'F': fusion beat, 'Q': unknown beat
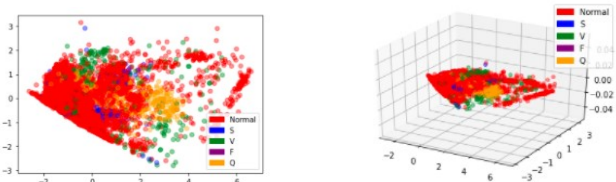

Graph 2: PCA on the Raw Data - MITBIH Dataset

PCA on the raw data does not reveal a very separable structure. This suggests simplet linear models would most likely not perform well on the raw data
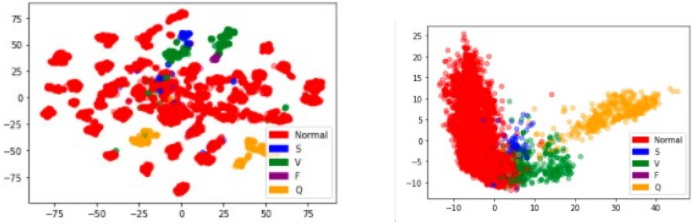

Graph 3: t-SNE on the Embeddings - MITBIH Dataset

$\mathrm{t}-\mathrm{SNE}$ on the embeddings shows a clear separation between classes, even for the rare F class The representation obtained from the baseline $\mathrm{CNN}$ reveals a much more separable structure. 

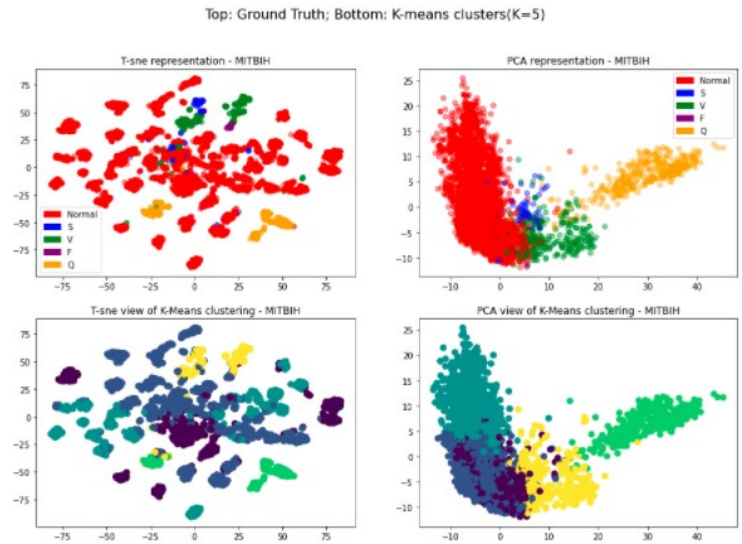

Graph 4: Attempt to Cluster with K-means on the Embeddings - MITBIH Dataset

Here we do train K-means on using the full training set on the embeddings produced by the baseline model.

What is shown is the prediction for the same subset of points as above, shown in the PCA and t-sne basis.

We observe that classes $\mathrm{Q}$ and $\mathrm{V}$ are very clearly clusterable. However, K-means seems to struggle in differentiating between class normal and S. This is understandable from either of the projections as these 2 classes seem to overlap the most.

\section{FOR PTBDB DATASET:}

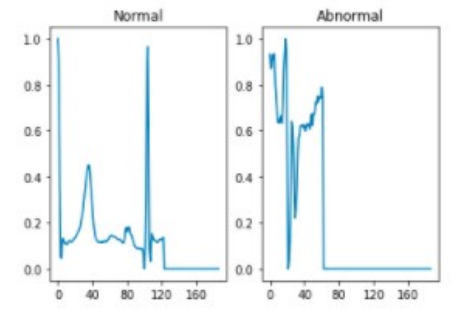

\section{Graph 5: Normal vs Abnormal Count}

Once more, quite unbalanced dataset, with abnormal having more than double the datapoints of normal

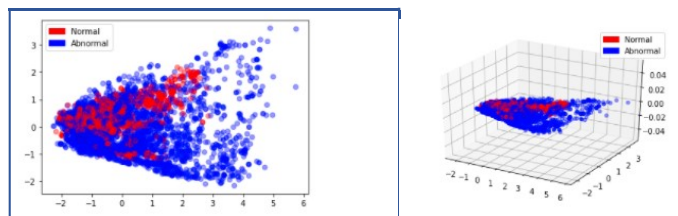

Graph 6: PCA on the Raw Data - PTBDB Dataset

PCA does not show such a clear separation.
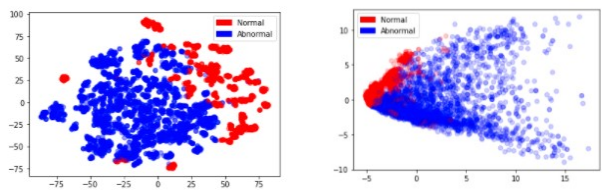

Graph 7: t-SNE on the Embeddings - PTBDB Dataset

But t-sne shows a much clearer separation between both classes. The PCA plot is not as clearly separable, with considerable overlap of both classes 


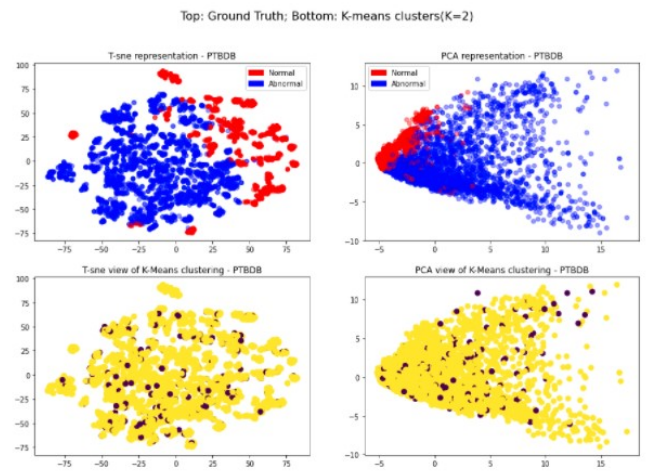

Graph 8: Attempt to Cluster with K-means on the Embeddings - PTBDB Dataset

\section{Conclusion and Future work}

A versatile 1-D Convolutional Neural Network $(\mathrm{CNN})$ is used for feature extraction and classification performed on two datasets MITBIH and PTBDB. We have done dimensionality reduction through PCA and tsne on both datasets and represented them visually in results and graphs section.

For future scope, we can check the variation in performance level for residual connection (Basic CNN) and Deep Residual CNN.

\section{References}

[1] Convolutional Neural Networks for Electrocardiogram Classification by Mohamad M. Alrahhal,Nassim Ammour https://www.researchgate.net/publication/324279944

[2] Deep Convolutional Neural Network Based ECG Classification System Using Information Fusion and OneHot Encoding Techniques by $\mathrm{Jia} \mathrm{Li}$, Yujuan $\mathrm{Si}$, Tao $\mathrm{Xu}, 3$ and Saibiao Jiang http://downloads.hindawi.com/journals/mpe/2018/7354081.pdf

[3] Full training convolutional neural network for ECG signals classification by Karboub Kaouter, Tabaa Mohamed, Dellagi Sofiene, Dandache Abbas, and Moutaouakkil Fouad

[4] Classification of ECG signals based on 1D convolution neural network by Dan Li ; Jianxin Zhang ; Qiang Zhang ; Xiaopeng Wei https://ieeexplore.ieee.org/document/8210784 